\title{
INSS Stage 4S
}

National Cancer Institute

\section{Source}

National Cancer Institute. INSS Stage 4S. NCI Thesaurus. Code C85422.

Patients are less than one year old with localized primary tumor and dissemination limited to the following specific anatomic sites: liver, skin, or bone marrow. 